\title{
Fragmentation des corps et des identités chez Chloé Delaume
}

\author{
Dawn Cornelio \\ University of Guelph
}

Le moi de l'homme n'est pas réductible à son identité vécue.

Jacques $\operatorname{Lacan}^{1}$

Comme le moi ne doit pas être réduit au vécu, l'autofiction ne doit pas être réduite à la vie. Toutefois, qui dit autofiction ne dit pas seulement fiction, et le vécu de l'écrivain ne peut pas être

\footnotetext{
${ }^{1}$ En 1948, lors du 11e Congrès des psychanalystes de langue française, dans un discours intitulé "L'agressivité en psychanalyse», Jacques Lacan a prononcé cette remarque.
} 
complètement évacué des textes. Dans un examen de n'importe quel aspect du travail de Chloé Delaume, la place du vécu est indéniable, car non seulement écrit-elle sa vie, mais elle vit son écriture aussi, sans permettre que l'une ou l'autre impose des limites. Particulier à l'autofiction de Delaume est un seul événement dramatique de sa vie qui figure fréquemment dans ses textes. La chercheuse qui entreprend une étude de ses œuvres doit décider quelle valeur accorder à l'événement autour duquel l'écrivaine construit son œuvre, son identité, sa vie, sa subjectivité. Car il serait faux de passer à côté de l'événement, tout comme il serait faux d'exagérer son rôle. En effet, l'auteure elle-même ne le nie pas, mais elle refuse de le laisser déterminer son identité personnelle. Alors, la chercheuse doit aussi l'inclure, mais elle doit peser ses mots quand elle parle, ou écrit, au sujet du moment qui a marqué la vie de la petite fille que l'écrivaine a été. Comment ne pas tomber, en cherchant à montrer de l'empathie, dans le mélodrame? Comment ne pas banaliser, par un ton qui se veut neutre et savant, une tragédie personnelle ? Comment ne pas s'approprier un événement qui ne peut appartenir qu'à l'auteure, la seule qui a le droit d'en parler dans son œuvre? De même, où placer la révélation dans une analyse ? Au début pour contextualiser? À la fin pour expliquer ou justifier une théorie? Dans une note en bas de page pour montrer qu'on sait, mais qu'on ne veut pas insister?

Peu nombreux sont ceux qui, pour peu qu'ils connaissent le travail de Delaume, ne sont pas au courant de l'événement que nous évoquons ainsi : le fait qu'en 1983, son père a tué sa femme (la mère de l'auteure), avant de se suicider, sous les yeux de leur fille, qui avait 10 ans à l'époque. Dans le présent essai, la stratégie privilégiée pour le traitement de cet 
événement ne sera pas de montrer son influence dans les textes de l'auteure, mais d'exposer ce qu'elle-même en fait dans certains textes, tout en l'entourant d'autres exemples qui permettront de voir de manière plus globale certains aspects de la production de cette écrivaine. Ce qui nous intéresse ici est donc rôle de la fragmentation - dans les descriptions du crime et dans d'autres cas aussi - dans la création d'une subjectivité active chez Delaume, car, dans sa tentative de prendre le contrôle d'une histoire qui lui a échappé et d'en construire une autre, elle procède par une mise en morceaux, c'est-à-dire par une fragmentation, sur laquelle elle opère une (re)construction. Nous proposons d'examiner la fragmentation sur deux plans : celui des corps - surtout celui des autres mais aussi celui de la narratrice - et celui de l'identité, particulièrement dans sa relation au nom - encore une fois, celle des autres et celle de l'auteure-narratrice.

\section{Fragmentations des corps}

Ouvrir au hasard une œuvre de Delaume implique de courir le risque de tomber sur un texte parsemé d'images violentes et sanglantes de corps humains assassinés, mutilés, cuisinés ou dépécés, peu importe qu'ils soient réels, fictifs, ou métaphoriques. Un coup d'œil sur plusieurs romans et récits nous permet de remarquer la généralisation du phénomène. Les Mouflettes d'Atropos (2000) propose un fantasme de la mise en morceaux du corps de la maîtresse du mari de la narratrice et de celui de nombreux hommes, représentants de leur sexe. Le Cri du sablier (2001) offre la description la plus explicite du 
meurtre-suicide des parents de l'auteure. Certainement pas (2004) se déroule dans le pavillon psychiatrique d'un grand hôpital et prétend vouloir trouver l'assassin du docteur Lenoir. Dans La Nuit je suis Buffy Summers (2007), les patients essaient de s'échapper d'un hôpital psychiatrique où l'infirmière fait prélever les organes des malades pour les vendre à ceux qui vont les consommer après avoir payé des prix exorbitants : «C'est donc très fermement qui Miss Mildred Ratched somme ses aides-soignants de prélever les organes d'usage, ainsi que les crânes et certains os [...]. Pour toute solde cette nuit, une seule satisfaction: le bocal tintinnabule ses cinq kilos de métacarpes, elle peut enfin honorer sa plus ancienne commande, le maire sera ravi » (p.5). Dans ma maison sous terre (2009) est situé dans un cimetière et se déclare une tentative d'assassiner la grand-mère de son auteure, tout en explorant le désir de la narratrice de participer à une séance de thanatologie. Une femme avec personne dedans (2011) prend comme point de départ le suicide d'une lectrice. Chez Chloé Delaume, le corps est découpé, segmenté, éclaté, nié, et remixé, suivant une expression chère à l'auteure.

La scène du meurtre-suicide, de la fragmentation des corps des parents est la plus explicite dans Le Cri du sablier, le troisième texte de l'auteure. La porte du roman s'ouvre avec celle que les policiers enfoncent en arrivant dans l'appartement à la suite du crime :

Les hommes nombreux forcèrent la porte. [...] Ils aspiraient chaque goutte pour se forcer à croire pour se forcer à dire j'y étais sans la peur sans le dégoût sans choc sans envier la crécelle de l'enfant moite d'A+. Ils salivaient chaque touffe de cervelle échevelée pour se forcer à croire se forcer à dire je suis venu pour vaincre et non pas pour regarder. Par-dessus la fine croûte de maman sur ma robe s'étala contiguë la mélassonne 
pitié le jus du parvenu la déjection des pleutres qui jalousent en geignant le clinamen aride qui s'abat sur tous ceux ornant faits divers. (Delaume, 2003a, p. $9^{2}$ )

Examiner cet extrait comme un ensemble montre non seulement une subversion de la syntaxe, mais aussi une fragmentation inattendue des phrases : le fait qu'elles ne sont pas fragmentées par la ponctuation attendue par le lecteur mène à une fragmentation de la pensée et de la compréhension (à part les points, tout au long du texte, il n'y a presque aucun signe de ponctuation qui découpe le texte en séquences maîtrisables). Comme le corps de chacun des parents décrit précédemment, le texte est à la fois un ensemble cohésif et un objet éclaté qui violente le lecteur. Comme le texte, le corps des parents est réduit à des fragments : les flaques et les gouttes apparaissent comme des euphémismes représentant le sang et tous les autres liquides vitaux qui s'échappent des corps et préparent les policiers et les lecteurs à la boucherie de la scène qui se matérialise sous leurs yeux. De même, il est possible de comprendre «l'enfant moite d'A+» comme un autre euphémisme pour le groupe sanguin du père, sans oublier que A est aussi l'initiale du nom de famille de cet homme qui, avant d'emménager du Liban en France, s'appelait Abdallah. Mais l'auteure ne se limite pas à évoquer les dégâts par des euphémismes, et ne tarde pas à frapper avec une violence ironique, en réduisant les corps parentaux aux «touffes de cervelle échevelée », à la " fine croûte de maman sur [s]a robe », $\mathrm{au}$ « jus du parvenu », le descripteur «parvenu » étant souvent lié au père. Les fragmentations des corps, dans une description tout aussi fragmentée, ainsi que la mise en évidence de ces

\footnotetext{
${ }^{2}$ J'incorpore une analyse alternative de ce texte dans mon article, «Elle se nomme Chloé Delaume : un parcours personnel et littéraire » (2014).
} 
fragments, représentent un élément fondamental et récurrent chez Delaume, qui semble devoir mettre en morceau, afin de voir ce qu'il y a à découvrir, avant de passer à la (re)construction.

De manière générale, le temps de narration delaumien est plutôt éclaté, et Le Cri du sablier n'échappe pas à cette tentative généralisée de déstabilisation des lecteurs. Ce n'est qu'une dizaine de pages plus tard que le lecteur du roman apprend ce qui a rendu nécessaire l'arrivée des policiers racontée plus haut : «En fin d'après-midi le père entra dans la cuisine tira à bout portant. La mère tomba première. Le père visa l'enfant. Le père se ravisa, posa genoux à terre et enfouit le canon tout au fond de sa gorge. Sur sa joue l'enfant reçut fragment cervelle » (Delaume, 2003a, p. 19; je souligne). Nous voyons le même événement, mais au moment même où il a lieu, quelques minutes avant l'irruption des policiers. Comme dans l'extrait précédent, les parties du corps des parents - et de la fille - se détachent de l'ensemble de l'être humain pour se disperser en liquides et en petits morceaux. Dans ces deux séquences, l'action se passe comme dans le vide : il manque toute sensation sauf l'impression visuelle de la violence : le lecteur n'entend pas les explosions qui auraient accompagné les coups de feu; il n'entend pas non plus les inspirations abruptes de la fille éclaboussée ni des policiers quand la porte s'ouvre sur la scène du meurtre; il n'entend pas les paroles des policiers, qui sont plus rapportées que prononcées quand ceux-ci se parlent. Dans ces scènes, le lecteur ne peut que voir chaque élément qui se détache, presqu'au ralenti, des corps réduits en parcelles séparées, qu'elles soient liquides ou solides, qui s'étalent et se dissipent. Cet extrait, avec le précédent, permet de remarquer que les composants des corps de chacun des parents ne 
touchent pas la fille de la même manière. C'est-à-dire les résidus de la mère n'arrivent pas jusqu'au corps de la fille et restent en "une fine croûte [...] sur [s]a robe», tandis que l'enfant reçoit un bout de cervelle du père sur la joue. Dans la mort, tout comme dans la vie, la présence de la mère est insuffisante à protéger la fille des violences du père ${ }^{3}$.

Désormais, la scène du meurtre-suicide n'est qu'un exemple de l'importance de la fragmentation corporelle dans l'écriture de Delaume. Par exemple, dans La Vanité des somnambules ainsi que dans Où le sang nous appelle, l'auteure met en scène un accident de voiture où la violence infligée aux corps des personnages parentaux permet à la narratrice de se séparer de l'histoire dont on pourrait penser qu'elle est la victime. Dans ces descriptions, la réalité qu'est la mort des parents passe par l'écriture et est ainsi contrôlée par la narratrice. Celle-ci ne subit pas la violence du crime, elle crée une histoire alternative qui l'écarte du sang et des bouts de chair. Dans La Vanité des somnambules, la mort violente des parents est d'abord réduite à une douzaine de mots : "Les Citroën ne sont pas fiables. 30 juin 83: autoroute et calcination » (p. 20). Ici, les corps ne sont ni dépiécés ni détaillés, mais leur destruction par la chaleur et le feu est tout de même anéantissante. Nous attirons l'attention sur cette courte description de l'accident, car une pareille scène surgit encore dans La Vanité des somnambules et aussi, une décennie plus tard, dans Où le sang nous appelle. En fait, seulement trois

\footnotetext{
3 Bien que, dans le cadre de cette étude, j'inclue l'analyse d'une scène de violence contre la fille aux mains du père, l'œuvre en est parcourue d'autres, qui montrent que, dans la plupart des cas, la mère, même si elle n'y participe pas directement, ne fait pas obstacle au père. Ces scènes devraient faire l'objet d'une analyse à part.
} 
chapitres plus tard dans le même récit, le passage de l'événement autobiographique à la mise en fiction se lit en toutes lettres dans une courte description de ce que la narratrice considère comme la création acceptable :

je suis orpheline loge fibreuse à cause d'un sale crime premier méninge oublions-le dure-mère scotomisons [...] un mensonge propre m'a proposé sillon central dans la BX familiale mes très chers parents ont cramé scissure de Rolando ça sentait le caoutchouc et scissure de Sylvius le poulet grillé lobe frontal c'est plus plausible il faut l'avouer lobe pariétal la fiction est la seule lobe temporal assainissant réalité lobe occipital affabulette à cérébrer gyrus cingulaire trauma initial : données effacées » (Delaume, 2003b, p. 45)

Dans cette citation, ce ne sont pas les corps des parents ni celui de leur fille qui se font dépiécer, mais plutôt le cerveau de l'auteure-narratrice qui travaille pour changer " un sale crime » en réalité acceptable, libre de honte, un fait divers plus admissible, plus convenable que celui où figurent les deux coups de feu. Séparés seulement par les mots représentant les pensées de cette même narratrice, les éléments du cerveau liés entre eux par l'emploi des lettres grasses dans le texte travaillent ensemble et triomphent, au moins momentanément, de la situation. Le cerveau, et le clavier, de la narratrice transforment le réel en histoire, mais l'auteure, par la présence $\mathrm{du}$ processus mental, ne manque pas de nous rappeler qu'il s'agit d'une autofiction, où l'écrivaine aurait « confié le langage d'une aventure à l'aventure du langage», comme l'a dit Doubrovsky.

Dans Où le sang nous appelle, l'accident de voiture fait encore une apparition, mais cette fois-ci, la description s'étend sur tout un chapitre d'une dizaine de pages, et le véhicule 
accidenté appartient à la famille de la sœur de la mère de l'auteure-narratrice, qui l'a accueillie après le décès de ses parents. Nous nous intéressons à cette scène d'accident comme aux autres parce qu'elle aussi est caractérisée par la fragmentation corporelle. Cette fois-ci, les éléments des corps une quarantaine au total, la plupart regroupés dans les quatre premières pages du chapitre - ne se détachent pas mais subissent néanmoins la violence du choc. Par ailleurs, dans l'épisode qui figure dans Où le sang nous appelle (surtout le chapitre 2, «En juin, probablement 1984 »), bien plus long que dans les scènes trouvées dans La Vanité des somnambules, il s'agit des parties du corps non seulement de la narratrice, mais aussi des autres membres de la famille, tout comme il s'agit des mains, des jambes, des dos, etc., et non seulement du cerveau réfléchissant. Malgré ces différences importantes, les épisodes permettent surtout d'attirer l'attention sur le fait que le corps humain occupe une place capitale dans la production delaumienne, et la présence des parties de corps n'est pas limitée au traitement d'un ou deux thèmes mais représente en effet un phénomène assez généralisé, comme nous venons de le voir, et comme nous le verrons encore ci-dessous.

Dans Les Mouflettes d'Atropos, la narratrice inflige une autre violence fictive à plusieurs de ses personnages : elle se venge de son mari, de celle que son mari lui a préférée et de ceux qui, dans le bar à hôtesses où elle se prostitue, la réduisent à un récipient sexuel, en s'attaquant dans chaque cas à leurs organes sexuels. Dans ses fantasmes, elle sert à son mari des recettes dont l'ingrédient principal vient des morceaux du corps de son amante. Lors de la scène la plus explicite, ce sont les lèvres du vagin qui seront servies, et, bien que ce ne soit pas le seul morceau du corps consommé, dans ce cas ce n'est ni la 
recette ni la dégustation qui joue le rôle principal, mais le geste amputatoire et violent qui raconte la séparation de la chair du corps encore vivant. Cette description est moins explosive que celle du meurtre-suicide, car la scène - un seul long paragraphe, en fait - se lit comme la transcription d'une intervention chirurgicale très contrôlée, organisée, et scientifique. Sont ici énumérés les parties de l'anatomie, les gestes de la praticienne et les instruments dont elle se sert : la litanie n'est interrompue que par les injonctions de la narratrice, qui somme l'amante de se taire et de se tenir tranquille. Il s'agit des petites lèvres du vagin, dont, selon la narratrice, l'élasticité facilite la prise, mais dont la petite taille oblige à un travail précis, car pour les servir au mari en tapas, il ne faut pas en perdre la moindre bouchée. Dans l'énumération des éléments du système reproductif de la femme, nous retrouvons donc les ovaires qui sont orangés, la vulve qui est ouverte sur l'Absolu, le col qui n'est pas marqué par une lettre de feu, et l'utérus dont les mycoses sont bénignes. Les gestes - saisir, tirer, ponctionner, révélation, dissection, radiation et épuration - nous conduisent, par un nombre presque égal de verbes et de noms, du début de l'intervention jusqu'à sa fin et sont accompagnés par la mention d'instruments médicaux tels que scalpel, coupelle, forceps et aiguille. Un seul outil non médical, le « Cutter » (Delaume, 2000, p. 31), surgit entre le forceps et l'aiguille et souligne la violence de l'intervention. Finalement, après la remarque «OPÉRATION TERMINÉE » (Delaume, 2000, p. 31), le dernier instrument demandé, Serviette, clôt le paragraphe et le passage. Chacun de ces objets est souligné dans le texte par l'emploi de lettres italiques et, avant le mot «Serviette » (Delaume, 2000, p. 31), la remarque "OPÉRATION TERMINÉE » attire l'attention du lecteur par ses petites majuscules encore plus criardes que l'unique 
majuscule employée pour commencer chaque étape de l'opération. Cette habitude typographique des écrits de Delaume sert non seulement à accentuer, mais aussi à briser la lecture et à forcer le lecteur à prêter une attention particulière aux expressions ainsi démarquées, ainsi qu'à s'interroger sur le choix de l'auteure des mots ainsi distingués; il s'agit donc de l'imposition d'une lecture fragmentée, car interrompue, qui se fait l'écho des parties du corps détachées de l'être humain en général, de la rivale de la narratrice dans cette scène. Les buts du passage sont multiples; à part s'acquitter d'une pulsion vengeresse et violente, contre l'amante et le mari aussi, la narratrice cherche, dans son excision et son exploration, une réponse à la question: pourquoi son mari a-t-il préféré ce vagin-là au sien? Toutefois, si le désir de représailles et de punition est satisfait par cette attaque médicalisée, la question reste sans réponse, l'investigation par excision est un échec, la mise en morceaux des organes génitaux n'a mené à aucune découverte, à aucune compréhension.

Dans ce récit, si la narratrice cherche une explication à sa situation de femme trompée seulement à l'intérieur du corps de l'amante, la pulsion vengeresse s'étend cependant à plusieurs corps masculins, surtout à l'aide de l'invention de la narratrice du « Bito-Extracteur $®$ (Brevet No. 4532XF/ 9785K/ Tous droits réservés) » (Delaume, 2003a, p. 58), un four à micro-ondes modifié qui permet l'introduction du pénis offensant, par une petite fenêtre aménagée à cette fin, avant de procéder à la cuisson de celui-ci, ce qui mènera à son détachement du corps. Dans le contexte de cet appareil, nous ne devons pas négliger de signaler la forte présence de références à l'Américaine Valerie Solanas par des citations, exactes et aussi faussées, du SCUM Manifesto, petit texte de 1967 dans lequel Solanas encourageait 
les femmes à trancher le pénis des hommes. ${ }^{4}$. L'appareil de Delaume, lié aux exhortations de Solanas (SCUM en anglais ici un acronyme de "Society for Cutting up Men », c'est-à-dire "Société pour la mise en morceaux des hommes») laisse imaginer une quantité extraordinaire de membres tranchés ; dans ce cas, l'appel littéraire et littéral de Solanas ainsi que le four transformé permettent au lecteur de procéder à sa propre accumulation imaginaire des corps dé-membrés, tout comme l'auteure le fait explicitement dans bien d'autres scènes, où les parties du corps se détachent et s'amassent de manière presque exponentielle et certainement déconcertante.

Tout comme dans la scène de dissection vaginale que nous venons de voir, ici aussi, la narratrice focalise son attention sur les parties génitales des individus visés pour punition. Bien qu'il ne s'agisse que d'une seule partie du corps, il est impossible de ne pas concevoir la possibilité de la répétition du geste qui s'accomplira par des moyens mécaniques, peut-être par une multitude de machines puisque les consignes détaillées pour la conversion du micro-ondes domestique en «Bito-Extracteur $®$ (Brevet No. 4532XF/ 978K/ Tous droits réservés) » (2000, p. 58) sont bien données dans le texte, et la narratrice encourage son lectorat à aussi se servir de sa propre créativité afin de détacher un maximum de pénis, car il faut « couper le mâle à la racine » (Delaume, 2000, p. 52). À la différence de l'excision de la femme, le démembrement des hommes ne cherche pas à apprendre quoi que ce soit à la narratrice; son seul but est punitif et pratique, car après avoir

\footnotetext{
${ }^{4}$ Pour une analyse approfondie du rôle de l'intertexte de Solanas dans Les Mouflettes d'Atropos, voir «Littéralisation et lignée féministe : l'héritage du Scum Manifesto dans Les Mouflettes d'Atropos de Chloé Delaume » (LedouxBeaugrand, 2013).
} 
été « branleuse » dans un bar à hôtesses, elle a trop vu de « ces bric-à-brac de queues putréfiées ces catalogues de difformations masculines ces piètres nomenclatures de virilités gerbantes de zgegs au quotidien où il ne manquait rien à l'inventaire que deux ou trois ratons laveurs pour faire de la poésie moderne que l'idée de faire le ménage [lui] est venue » (Delaume 2000, p. 54).

Dans l'ensemble de textes signés Chloé Delaume, le dépeçage des corps ne se limite pas aux corps vivants ou saisis au moment de leur mort, mais il s'étend aussi à des descriptions de corps dans leur tombe, après la mort, et encore une fois, les parties des corps - les os, les organes, les chairs - sont détachées de l'ensemble, au moins dans les descriptions. Dans Dans ma maison sous terre, l'auteure peint d'abord l'image générale de corps en décomposition, pour en arriver à la fin à ceux de sa mère et de son grand-père maternel :

On ne vérifie pas à quoi ressemblent les morts une fois qu'ils sont rangés. On ne vérifie pas l'apparition de la tache verte au niveau des fosses iliaques, son extension qui finit par gagner progressivement tout l'abdomen. On ne vérifie pas la modification de la flore intestinale, la prolifération des mycètes, les altérations progressives du substrat. [...] La cirrhose de papi qui putréfie ruisselante sur les os de maman, ça doit rester secret. (p. $7-8$; je souligne)

De même, vers la fin d'Où le sang nous appelle, l'auteurenarratrice se trouve, au Liban, devant la tombe de son père, accomplissant un des buts du voyage dans ce pays qui constitue le sujet principal du texte. Le face à face avec la tombe du père se raconte par un monologue dialogué avec le père lui-même, dans lequel, sans constituer une partie importante, des questions sur l'état du corps ne sauraient se taire : «Allez, papa, 
raconte. Les morceaux de ta tête, ton cadavre acéphale, quand ils ont atterri sur les os de ton père, les mètres cubes compacts de fange racornie faite de tellement d'aïeux, ils t'ont dit bienvenue ou ils t'ont engueulé ? Quelques années après, elle t'a dit quoi ta mère, tout le temps où elle te pourrit dessus? » (p. 347-348; je souligne). En faisant abstraction de l'aspect répugnant de la décomposition des corps qui est souligné par les références à la pourriture, on ouvre la possibilité de noter un autre thème présent dans ces deux passages : la famille. Dans les deux passages, l'aspect familial du caveau est souligné - dans le cas de la mère de l'auteure, son propre père est mentionné explicitement et, dans le cas du père de Delaume, celui-ci rencontre, dans la tombe, non seulement son père, mais aussi sa mère, ainsi que des générations d'aïeux qui l'ont précédé dans ce lieu. Dans les deux cas, bien que les éléments $\mathrm{du}$ corps soient manquants (on pense à l'image du " cadavre acéphale ») ou détachés («les morceaux de ta tête », «les os de ton père »), d'une manière plus générale, la tombe est un lieu de mise ensemble à l'échelle plus grande: les membres des familles, détachés de celle-ci par la mort (ou par la vie, selon la perspective) sont réunis dans un ensemble plus vaste que les corps des individus. Ils s'y retrouvent dans un état de décomposition qui les intègre de plus en plus les uns aux autres et empêche même leur séparation en pièces détachées. Ces images se font l'écho de la hantise de la fille, en qui s'est infiltré le père par son sang et ses morceaux de tête éclaboussant sa descendante. Le détachement dans la tombe, comme dans la description du meurtre-suicide, mène à une mise ensemble des membres de la famille. C'est-à-dire, tout comme la fille se trouve éclaboussée par les chairs et le sang de son père au moment de son crime, dans la tombe, les parties des corps des 
différents membres de la famille se mélangent les unes aux autres ; ainsi, le détachement de son propre corps mène à une meilleure intégration dans le groupe familial. Dans la mort, le corps perd certes son intégralité, mais par le détachement des éléments, l'union avec d'autres corps familiaux est facilitée. Le désir d'union corporelle après la mort est explicité par la narratrice au tout début de Dans ma maison sous la terre, quand elle parle de la tombe où se trouvent les cadavres de sa mère et de son grand-père : « Il reste une troisième place. [...] Je voulais la rejoindre, j'explique à Théophile, je voulais être contre, déjouer l'ordre établi. [...] Sûrement pour la toucher. Encore et malgré tout. Un dernier rapprochement, ultime et terminal [...]. » (p. 8) Bien que, lors du meurtre-suicide, le sang et la chair de la mère ne touchent pas la fille de la même manière que ceux du père, nous voyons ici, chez la narratrice, le désir de sentir son cadavre entrer en contact intime avec celui de sa mère, d'éliminer donc la séparation imposée par le père.

Bien que, jusqu'ici, notre analyse se soit limitée aux corps d'autres personnes, il ne faudrait pas passer sous silence que celui de Chloé Delaume, personnage de fiction, a connu une enfance marquée par l'abus corporel et psychique antérieur au crime du père. Un épisode révélateur de la brutalité de l'enfance de la narratrice raconte des vacances en camping à Toulon où la fille, pendant l'absence des parents, a été enfermée à l'intérieur de leur tente sous la chaleur écrasante d'une après-midi d'été. Habillée en jeans, malgré la température, pour cacher les traces des coups déjà reçus, la fille ne peut pas quitter la tente pour se mettre à l'ombre sous un arbre, ni s'asperger le visage au bloc sanitaire, puisqu' " une épingle à nourrice retient la fermeture Eclair [de la tente] de l'extérieur» (Delaume, 2003a, p. 37). La fille de huit ans qui subit la chaleur, réduit la réaction de son 
corps à sa transpiration, qu'elle compare au beurre ou à la margarine, et essaye de mettre fin à sa souffrance généralisée en s'étouffant. Dans les deux courtes phrases qui décrivent cette tentative figurent sept éléments du corps: "Elle vide ses poumons se pince le nez et colle la main sur sa bouche. Coince ses lèvres entre l'annulaire et le majeur pour plus de précaution» (Delaume, 2003a, p. 39 ; je souligne). Mais quoi qu'elle fasse aux différentes parties du corps, elle ne réussit pas à s'empêcher de vivre et, au retour du père, la rage de celui-ci, offensé par l'odeur de transpiration, le pousse à lui infliger une nouvelle violence inattendue: «[l]e père revient bientôt déroulant derrière lui un tuyau d'arrosage. Le jet glacé abrupt violace lèvres et veinures la giclade déversée sur le sommet du crâne tentacules acérées pénétrant jusqu'aux tempes, ça palpite en cristaux sang se fige sinus moelle muqueuse chair et os [...] » (Delaume, 2003a, p. 41 ; je souligne). Face à cette attaque, et de manière encore plus abrupte que dans la tentative de suicide par étouffement, les parties du corps - les lèvres, les veinures, le sommet du crâne, les tempes, le sang, les sinus, la moelle, la muqueuse, la chair et les os, et plus loin encore, le cœur, les doigts - se dissocient de l'ensemble du corps, dans la souffrance et le martyre. La violence n'a pas seulement pour effet de faire souffrir l'être physique, elle le fait voler en éclats, se désintégrer en une série de pièces détachées. Cette désintégration créée par l'énumération donne l'impression d'une plus grande peine chez l'enfant que lorsque c'est son corps en entier qui éprouve les douleurs causées par la chaleur ou par l'eau froide censée l'aider non pas à se rafraîchir, mais à se nettoyer et à éliminer l'odeur de sueur... 


\section{Fragmentation de l'identité}

Chez Delaume, la fragmentation de l'identité se manifeste souvent à travers l'emploi du nom. Dans une analyse du rôle du nom dans sa production, il faut se souvenir que ce n'est pas seulement l'auteure qui a changé de nom en décidant de s'appeler et de se faire appeler Chloé Delaume, mais toute la famille a dû changer de nom quand elle a décidé de réintégrer la France après quelques années passées au Liban dans la famille du père de l'auteure. Bien que la fillette n'ait pas eu de rôle à jouer dans la décision d'adopter une nouvelle identité onomastique, pas plus qu'elle n'a pu choisir celle-ci, le changement de nom revêt une importance particulière et figure dans plusieurs textes de Delaume. Dans son dernier ouvrage, Où le sang nous appelle, signé par Delaume et son conjoint actuel, le journaliste Daniel Schneidermann, la relation du changement de nom est succincte et directe :

Les mots n'ont plus pour sens que ceux qu'on leur impose, les noms c'est la même chose. Prénoms et patronymes se fabriquent et s'imposent, ciseaux consonnes colle, voyelles. C'est 1986, en France. Elle a treize ans et demi, elle s'appelle Nathalie. De sa naissance à ses six ans, c'était Nathalie Abdallah. Son père Selim, sa mère Soizic ${ }^{5}$. Ensuite, ils ont changé. Selim Abdallah pour Sylvain Dalain. La mère voulait Sacha, mais Sacha ce n'est pas français, Sacha Distel lui-même est russe, la préfecture ne peut valider. Sylvain, de silvanus, l'homme des bois, des forêts. (p. 116)

Le court extrait montre non seulement une partie du défilé des noms «officiels», ceux qui seront inscrits dans un

5 En lisant ce dernier texte de Delaume, je me suis rendu compte que l'orthographe du prénom de la mère a changé : par exemple, dans Dans ma maison sous terre, ce prénom ne s'écrit pas «Soizic » mais «Soazick» (p. 20). Les différentes orthographes restent à être examinées et analysées. 
livret de famille, mais aussi la nature aléatoire des choix. Il n'y a pas trop d'évidence d'une tentative de connexion, de sens ou de filiation, seulement un peu de volonté de francisation derrière les noms choisis. Ces derniers sont le résultat d'un travail de bricolage : des coups de ciseaux éliminent les consonnes trop arabes et la colle permet d'y ajouter plus de voyelles bien françaises. Mais même face à la volonté, aussi aléatoire soit-elle, il y des contraintes - le choix maternel pour le prénom du père, Sacha, ne peut être retenu, car il n'est pas assez français, selon l'autorité de la République représentée par la préfecture. La femme n'a pas le pouvoir d'octroyer le (pré)nom du mari. C'est le cas contraire quand Delaume choisit le nom sous lequel elle vit et écrit ; comme nous verrons ci-dessous, son choix était très explicite et délibéré.

Si la fille, non plus, ne peut réellement changer le nom de son père, elle trouve comme elle peut des manières de manipuler l'appellation, le rôle et même l'existence de l'homme: «Mon père ne doit être Sylvain que pour moi, pour les autres il doit être ton, son, le père. Je dépouille l'homme de son prénom, plus personne ne pourra le nommer, alors il ira en enfer. J'applique les châtiments que je peux » (Delaume, 2009, p. 47). « Je dépouille » : le choix de verbe est déterminant, car il permet de faire le lien entre la dépossession du nom et le démembrement physique vu plus haut, étant donné que l'enlèvement de la peau à un animal est le premier sens donné dans le dictionnaire, tandis que la privation d'une possession en est le cinquième (larousse.fr). Sans mentionner la relation avec la mort qui vient du fait que le verbe conjugué à la première personne du singulier se prononce comme le nom, la dépouille. Il s'agit ici d'un double exemple de la fragmentation delaumienne: au premier degré, la narratrice se donne le 
pouvoir de diviser les identités de son père (Sylvain pour elle ; père pour les autres) ; au deuxième degré, le verbe dépouiller nous ramène au concret, au physique, au corps associé aux identités.

Tout comme ses parents ont tenté de couper, en changeant de nom, non seulement l'origine arabe du père mais aussi sa relation familiale au terroriste Georges Ibrahim Abdallah, Nathalie Dalain a changé de nom pour se créer une identité indépendante de sa famille et de son histoire. À l'opposé des choix de ses parents, son choix n'a rien d'aléatoire :

Je m'appelle prénom nom bien plus qu'un pseudonyme. Le choix des référents m'a vomi parrainage, ce fut un kidnapping. J'ai imposé Chloé cancer du nénuphar ; extrait l'arve de l'aume pour toujours me trouver de l'autre côté du miroir. Reconfiguration jusqu'à mon ombilic, se détacher des limbes, oui, une bonne fois pour toutes. Boris Vian se débattait, Artaud aussi, je me souviens. Ils se refusaient sec à m'engendrer, je sais. Je les ai violentés : née de père et de père. Je me suis moimême engrossée. (Delaume, 2010, p. 5-6)

D'une manière définitive et surtout active - six différents verbes conjugués à la première personne du singulier font avancer l'extrait -, l'auteure choisit non seulement son nom, mais aussi ses géniteurs, qu'ils soient d'accord ou pas. D'un accouplement violent, Chloé Delaume barre l'existence de Nathalie Dalain et recommence à zéro, naissant de père et de père, choix littéraires qu'elle trouve des plus convenables. Mais le geste, malgré sa force, malgré le désir qui le motive, ne suffit pas : Dalain n'a pas évacué la vie de Delaume. À la conclusion de La Vanité des somnambules, Nathalie réussit à chasser Chloé de son corps. Même, plus tard, quand Chloé réussira la prise de contrôle du corps, Nathalie ne sera pas effacée : "Je n'habite pas mon corps, j'ose à peine l'habiter, parce qu'il n'est pas le 
mien, mais celui de Nathalie. De Nathalie Dalain, fille de Soazick et d'on ne sait pas bien qui. Les démarches administratives m'effraient, alors tout ce qui valide mon existence sociale est encore à son nom. Son nom à elle, son nom de petite fille » (Delaume, 2009, p. 202), car elle s'appelle encore et toujours "Nathalie, Anne, Hanné, Suzanne Dalain à l'état civil» (Delaume, 2009, p. 202). L'existence de Nathalie est toujours sous la surface de la vie de Chloé, que ce soit par les souvenirs ou, plus concrètement, par les documents administratifs: la tentative de remplacement se réduit donc à un autre exemple de fragmentation.

Ce n'est qu'avec la publication de 2013, Où le sang nous appelle, que la narratrice abordera la possibilité de rayer ellemême Nathalie Dalain de l'état civil pour la faire remplacer par Chloé Delaume: «Elle est loin, tu comprends, plus elle s'approche de moi plus elle touche à sa fin, elle n'existe plus, tu sais, Nathalie. Un avocat s'occupe de l'effacer de mes papiers, je suis Chloé Delaume » (p. 134). Il paraîtrait donc que Dalain sera définitivement éloignée des papiers de Delaume, mais il reste à voir si cette démarche réussira mieux que celle des parents, qui cherchaient à contourner le patronyme arabe, car la famille des hébergeurs n'a jamais oublié que Nathalie était en partie arabe et toujours fille de son père : "Lorsque la chambre est en désordre, le père déclare : Dis-moi, Véro, c'est pas à $50 \%$ qu'elle l'est, c'est à $80 \%$ au bas mot. Cette statistique durant six ans tiendra lieu pour eux de leitmotiv» (Delaume, 2013, p. 106); "folle comme son père tordue comme lui [...]. [...] Folle comme son père tarée comme lui » (Delaume, 2009, p. 69 ; en italiques dans le texte); "Nathalie tu penses bien qu'on ne l'aurait pas prise si elle s'était appelée Adballah» (Delaume, 2013, p. 121 ; en italiques dans le texte). L'écrivaine elle-même ne peut que 
rester consciente de son ascendance, mais au-delà de la relation génétique partagée entre tous les parents et enfants - «[1]ien du sang bien touillé folie en héritage » (Delaume, 2003a, p. 73) -, chez la narratrice, il y a aussi la peur d'être souillée par le sang du père qui est tombé sur elle lors de son suicide : « [e]n explosant le père par sa tête morcelée trouva encore moyen de ramper au dedans. [...] Ton sang séché papa qui virevolte et s'engouffre voulait tant pénétrer l'intérieur et vrombir égratigner les cols et les plaies intérieures » (Delaume, 2003a, p. 25). Le sang du père est bien sûr déjà dans le corps de la fille, mais avec ces dernières observations s'ouvre la possibilité d'une double interprétation : au moment du meurtre-suicide, ce sang peut aussi passer dans l'autre corps par la peau, par des ouvertures dans la peau, par les oreilles dans le vrombissement, ou, à un niveau plus psychologique, il peut entrer par les yeux, qui ne pourront jamais oublier ce qu'ils ont vu. Malgré les changements de nom, le sang paternel - que ce soit par-dedans ou par-dehors, par la naissance ou en la forçant à devenir témoin de son crime - aura infiltré sa fille.

Aux côtés des noms proprement dits de Nathalie Dalain et de Chloé Delaume, et aussi aux côtés de maints surnoms, gentils ou insultants (L'Enfant, La Petite, La Connasse), imposés de l'extérieur, apparaissent d'autres appellations choisies par l'auteure, et ce, depuis un jeune âge : «Elle a treize ans et demi, au dedans s'appelle Nini, parfois Super Nini, et jusqu'ici, à tout, elle aura résisté. Elle s'appelle Nathalie, généralement, ailleurs. » (Delaume, 2013, p. 118) Les rôles et les identités fusent de tous les domaines, de tous les contextes, allant, dans son premier récit, de la combinaison de la haute culture (Atropos) avec la culture populaire (mouflette), "Je suis la mouflette d'Atropos» (Delaume, 2000, p.68), jusque dans 
l'avant-dernier, qui fait l'inventaire en cataloguant un échantillon nombreux, mais sans doute incomplet :

Je suis Chloé Delaume, auteur, narratrice, héroïne. Je suis aussi la Reine, sa majesté exige qu'il y ait du répondant, une collectivité, pas juste des pseudonymes, des habits et des masques, partager les pouvoirs, désirs et savoir-faire. Je suis Clotilde Mélisse et peut-être Mélissandre, il faudra me baptiser en légion, je suis légion, chaque voix a sa fonction, la Petite, la Connasse, la Patronne, Carapace, Égérie et Sibylle. La Tueuse, la Régisseuse, Mademoiselle Souffreteuse, Princesse Panique, Poudreuse, Collapse et Agonie. (Delaume, 2012, p. 132)

Delaume elle-même, en parlant de son œuvre, commente la multitude d'identités auxquelles elle s'associe: "Car contrairement à ce qu'on croit parfois, je ne suis pas schizophrène, mais j'ai un véritable problème de dissociation, très net, oui. [0]n est plusieurs quand je me pose sur une chaise, tu vois? [...] Mais l'écriture n'est pas thérapeutique: je crois qu'on ne guérit pas. Comme on ne fait pas une analyse pour guérir, mais pour être au plus près de sa parole et de soimême » (Leménager, 2012, sp). Les identités, tout simplement dit, sont des choix qui peuvent se comprendre comme des prises de contrôle par le mode de la littérature. «Pour retrouver la parole, inventer les mots de l'histoire, elle devient écrivain. La petite Nini écrit, devient un peu pute, se re-nomme Chloé Delaume, est publiée dans des revues d'avant-garde, milite dans des groupes politico-littéraires qui veulent bouleverser le monde et la littérature, se suicide, se re-milite, se marie, se re-marie, se rate avec constance.» (Delaume, 2013, p. 51) L'emploi d'une série de verbes pronominaux - cinq au total, faisant partie d'un ensemble tout en étant détachés les uns des autres par les virgules, tout comme, chez l'auteure, les noms et identités distincts, tout comme les éléments d'un corps 
dépecé - souligne le désir delaumien de prendre le contrôle de sa vie, d'écrire sa propre histoire, d'«imposer un second commencement. Où la fiction se mêle à la vie, où le réel se plie aux contours de $m a$ fable. Celle que j'écris chaque jour, dont je suis l'héroïne. Mon ancien Je par d'autres se voyant rédigé, personnage secondaire d'un roman familial et figurante passive de la fiction collective » (Delaume, 2010, p. 6).

Comment alors intégrer la notion de fragmentation qu'elle soit du corps, du nom, ou de l'identité - à une œuvre qui se proclame être une construction personnelle et délibérée ? La réponse se trouve dans la métaphore que Delaume utilise pour la description de sa pratique: celle du laboratoire. Le laboratoire, c'est l'endroit où les éléments distincts peuvent être détachés d'un ensemble préexistant, pour être mélangés et mixés ensemble, afin de produire quelque chose de nouveau, d'inattendu : « [l]'autofiction est désormais l'ultime laboratoire : le laboratoire de la déconstruction, de la dissémination, de la prolifération folle des Je. Mais ce laboratoire n'est pas celui d'un savant fou : les expériences qui y sont menées portent bien audelà de la littérature » (Delaume, 2010, 4e de couverture). La réduction en matières premières des corps et des identités est à la base de la création autofictionnelle sur laquelle l'auteurenarratrice-héroïne Chloé Delaume construit son œuvre.

\section{Bibliographie}

CoRnelio, Dawn. (2014), «Elle se nomme Chloé Delaume : un parcours personnel et littéraire », dans Adrienne Angelo et Erika Fulop (dir.), Protean Selves: First-Person Voices in 
Twenty-First-Century French and Francophone Narratives, Newcastle-upon-Tyne, Cambridge Scholar's Press, p. 40-53.

Delaume, Chloé. (2000), Les Mouflettes d'Atropos, Paris, Farrago.

-. (2003a [2001]), Le Cri du sablier, Paris, Gallimard, coll. « Folio ».

—. (2003b), La Vanité des somnambules, Paris, Farrago/Léo Sheer.

—. (2004), Certainement pas, Paris, Éditions Verticales.

—. (2007), La Nuit je suis Buffy Summers, Paris, Éditions Ère.

—. (2009), Dans ma maison sous terre, Paris, Seuil, coll. « Fiction $\&$ Co ».

—. (2010), La Règle du je, Paris, PUF, coll. « Travaux pratiques ».

-. (2012), Une femme avec personne dedans, Paris, Seuil, coll. «Fiction \& Co ».

- et Daniel SchneIDERMANn. (2013), Où le sang nous appelle, Paris, Éditions du Seuil, coll. « Fiction \& Co ».

Doubrovsky, Serge. (1977), Fils, Paris, Éditions Galilée.

LEDOUX-BEAUGRAND, Evelyne. (2013), «Littéralisation et lignée féministe : l'héritage du Scum Manifesto dans Les Mouflettes d'Atropos de Chloé Delaume», Nottingham French Studies, vol. 52, no 3, automne, p. 337-349.

LEMÉNAGER, Grégoire. (2012), «Castillon-Delaume : Ni prudes, ni soumises », entretien avec Claire Castillon et Chloé Delaume, Le Nouvel observateur, 29 janvier, <http://bibliobs.nouvelobs.com/romans/20120118.0BS909 5/castillon-delaume-ni-prudes-ni-soumises.html>. 


\title{
Résumé
}

Cette étude traite du rôle de la fragmentation dans la création d'une subjectivité active chez Chloé Delaume. Dans sa tentative de prendre le contrôle d'une histoire familiale qui lui a échappé et d'en construire une autre, l'auteure procède par une mise en morceaux, c'est-à-dire par une fragmentation, sur laquelle elle opère une (re)construction. Nous proposons d'examiner la fragmentation sur deux plans : celui des corps - surtout celui des autres mais aussi celui de la narratrice delaumienne - et celui de l'identité personnelle, particulièrement dans sa relation au nom - encore une fois, celle des autres individus et celle de l'auteure-narratrice.

\begin{abstract}
This study deals with the role of fragmentation in creating an active subjectivity in the works of Chloé Delaume. Attempting to take control of a family story that got away from her and create her own story, the author first to breaks things to pieces - in other words, into fragments - which she uses to (re)construct something different. The notion of fragmentation is considered here on two levels: fragmentation of bodies whether they belong others or to Delaume's narrator, and fragmentation of personal identities, particularly as they relate to names - either the names of others or of the author-narrator.
\end{abstract}

